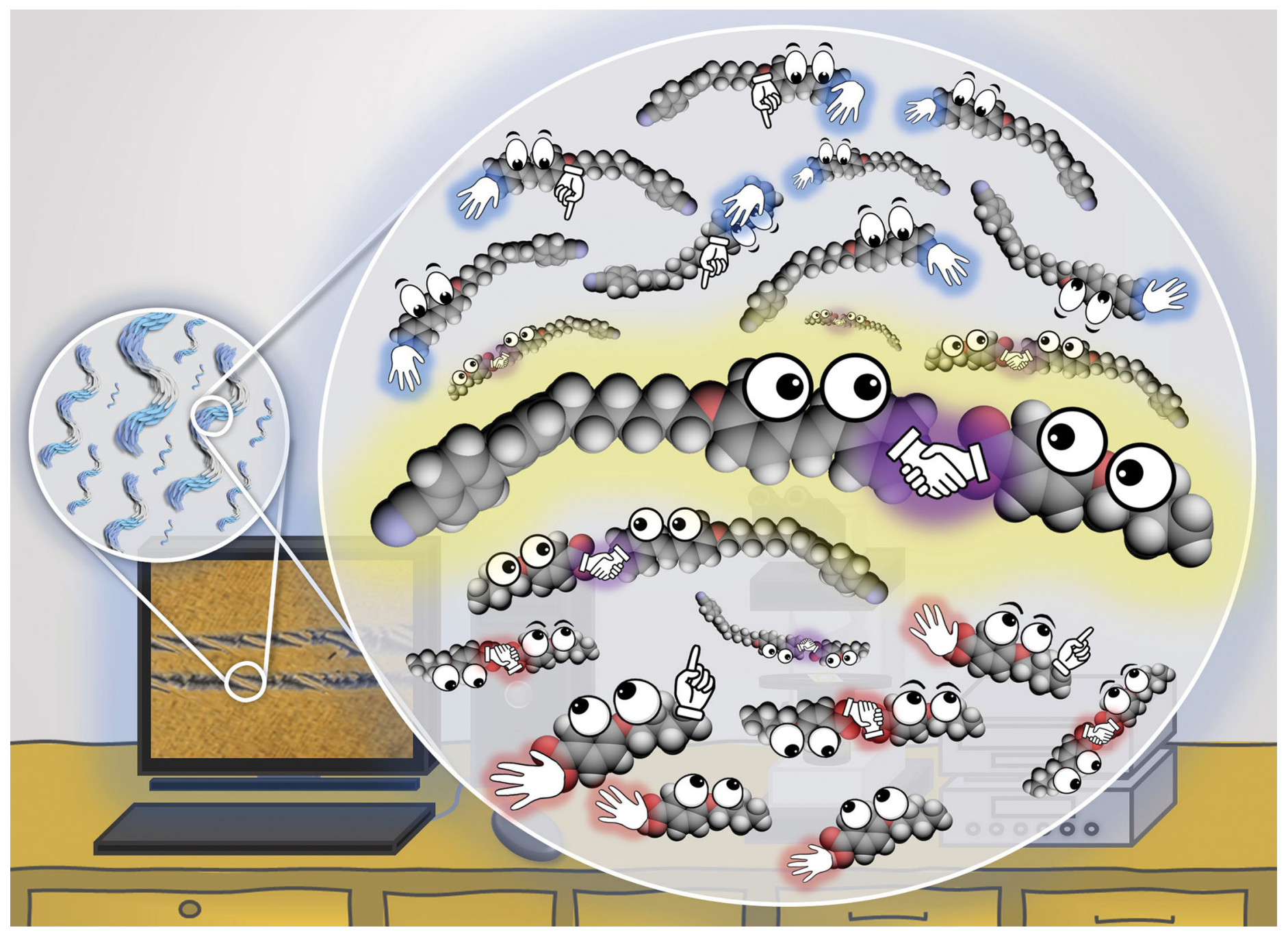

Showcasing research from Professor Corrie Imrie's liquid crystal synthesis laboratory, Department of Chemistry, School of Natural and Computing Sciences, University of Aberdeen, UK.

Supramolecular liquid crystals exhibiting a chiral twist-bend nematic phase

The helicoidal twist-bend nematic, $N_{T B}$, phase of liquid crystal is fascinating for a number of reasons, particularly because it is the first example of spontaneous chiral symmetry breaking in a fluid with no spatial ordering. An intriguing question is how the structural chirality of the $\mathrm{N}_{\text {TB }}$ phase responds to intrinsic molecular chirality; the chiral twist-bend nematic phase, $\mathrm{N}^{*}{ }_{\mathrm{TB}}$ has been observed in only a handful of materials. This work describes the first examples of the $\mathrm{N}^{*}{ }_{\mathrm{TB}}$ phase exhibited by supramolecular liquid crystals formed by hydrogen-bonding through molecular recognition between unlike $\mathrm{H}$-bonded donor and acceptor fragments.
As featured in:

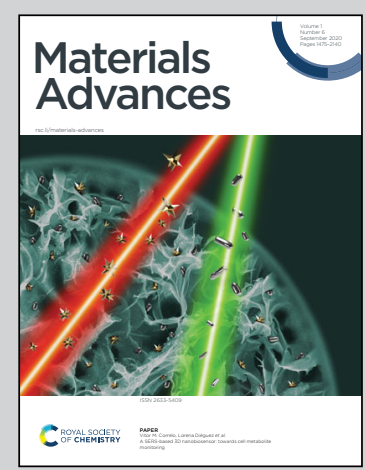

See Rebecca Walker et al., Mater. Adv., 2020, 1, 1622. 
Check for updates

Cite this: Mater. Adv., 2020 1,1622

Received 12th May 2020, Accepted 1st July 2020

DOI: 10.1039/d0ma00302f

rsc.li/materials-advances

\title{
Supramolecular liquid crystals exhibiting a chiral twist-bend nematic phase $\dagger$
}

\author{
Rebecca Walker, (D) *a Damian Pociecha, (D) ${ }^{\mathrm{b}}$ Mirosław Salamończyk, (D) ${ }^{\mathrm{b}}$ \\ John M. D. Storey, ${ }^{a}$ Ewa Gorecka (D) $^{\text {b }}$ and Corrie T. Imrie (D) ${ }^{a}$
}

\begin{abstract}
A selection of novel, chiral supramolecular liquid crystals formed by hydrogen-bonding are reported and their phase properties characterised by polarised optical microscopy, X-ray diffraction and resonant soft $X$-ray diffraction. Their behaviour is compared to that of their achiral analogues. The binary mixture of the bent-shaped, non-mesogenic $\mathrm{H}$-bond acceptor 1OB6OS and chiral $\mathrm{H}$-bond donor (S)-(2-Me)4OBA exhibits chiral nematic $\left(N^{*}\right)$ and chiral smectic $A\left(S m A^{*}\right)$ phases. Doping the twist-bend nematogenic $\left(\mathrm{N}_{\mathrm{TB}}\right)$ complex 1OB6OS:4OBA with $(\mathrm{S})-(2-\mathrm{Me}) 4 \mathrm{OBA}$ in ternary mixtures induces a transition from the $N^{*}$ phase to the chiral twist-bend nematic phase $N^{*}{ }_{T B}$, the first example seen in a supramolecular liquid crystal. The binary mixture of CB6OS and (S)-(2-Me)4OBA exhibits $N^{*}$ and $N^{*}$ TB phases, with the helical structure of the latter having a periodicity of approximately 2 complex lengths $(\sim 8 \mathrm{~nm})$. The nature of the hydrogen bond is reversed in a binary mixture of the bent-shaped, twist-bend nematogen CB6OBA with $(S)-(2-M e) 4 O B A$, and this exhibits the phase sequence $N^{*}-N^{*}{ }_{T B}-S m C *-S m X$.
\end{abstract}

\section{Introduction}

The twist-bend nematic phase, $\mathrm{N}_{\mathrm{TB}}$, has been a topic of very significant interest for the liquid crystal community since its experimental discovery in $2011,{ }^{1}$ many years after its initial prediction by Meyer in $1976^{2}$ and later independently by Dozov in $2001 .^{3}$ Unlike the chiral nematic (cholesteric) phase, in which the director twists around an axis perpendicular to the long axes of the molecules, the director in the $\mathrm{N}_{\mathrm{TB}}$ phase is tilted at a constant angle $\theta<90^{\circ}$ with respect to the helix axis and precesses on a cone forming an oblique helix of pitch about two orders of magnitude shorter than that typically found in the $\mathrm{N}^{*}$ phase, and equal to just a few molecular lengths. ${ }^{4}$ The $\mathrm{N}_{\mathrm{Tв}}$ phase can be considered as a generalised case of the chiral nematic phase and has been described as the 'structural link' between the $\mathrm{N}$ and $\mathrm{N}^{*}$ phases. ${ }^{4}$ Molecular curvature is widely considered to be the essential prerequisite for the formation of the $\mathrm{N}_{\mathrm{TB}}$ phase and the vast majority of twist-bend nematogens are odd-membered liquid crystal dimers (see, for example ref. 5-12). Undoubtedly the most fascinating feature of the $\mathrm{N}_{\mathrm{TB}}$ phase is that its chiral superstructure is formed despite its constituent molecules being chemically achiral, and indeed the

\footnotetext{
${ }^{a}$ Department of Chemistry, School of Natural and Computing Sciences, University of Aberdeen, Meston Building, Aberdeen AB24 3UE, UK. E-mail: rebecca.walker@abdn.ac.uk

${ }^{b}$ Faculty of Chemistry, University of Warsaw, ul. Zwirki $i$ Wigury 101, 02-089 Warsaw, Poland

$\dagger$ Electronic supplementary information (ESI) available. See DOI: 10.1039/ d0ma00302f
}

$\mathrm{N}_{\text {Тв }}$ phase represents the first example of such spontaneous chiral symmetry breaking in a fluid system with no long-range positional order. As the induction of chirality is spontaneous, there exists an equal probability of the formation of either handedness of helix and hence the $\mathrm{N}_{\mathrm{TB}}$ phase consists of doubly degenerate locally chiral domains of opposite handedness, giving a globally achiral (racemic) phase. This raises the intriguing question of how the $\mathrm{N}_{\mathrm{TB}}$ phase, having spontaneous structural chirality, will respond at a microscopic level to the presence of molecular chirality. This has been theoretically addressed by Meyer, who extended the elastic instability model of the $\mathrm{N}_{\mathrm{TB}}$ phase to consider the effect of a chiral field introduced by chiral doping. ${ }^{13}$ This model suggests the removal of the double degeneracy of the chiral domains, with the new ground state of the $\mathrm{N}_{\text {тв }}$ phase being the domains with the same handedness as the dopant. With increasing chiral doping, the conical angle in this favoured helical state increases, while that in the unfavoured helical state decreases and eventually the more favoured handedness dominates. The helical pitch is essentially unchanged. Thus, chiral doping is predicted to increase the stability of the $\mathrm{N}_{\mathrm{TB}}$ phase. Another theoretical model including the effects of molecular chirality was described by Longa and Tomczyk, who used minimum coupling Landau-de Gennes theory to reveal the possibility of a polar, chiral $\mathrm{N}_{\text {тв }}$ phase. ${ }^{14}$ At present, only a handful of systems exhibiting the transition between chiral nematic and twistbend nematic phases have been observed experimentally. These have included mixtures of twist-bend nematogens with a chiral dopant ${ }^{15,16}$ as well as intrinsically chiral materials, with 
the latter being particularly rare. ${ }^{17,18}$ Recently, we reported the first comparison between racemic and chiral versions of the same twist-bend nematogens and found that $T_{\mathrm{N}\left({ }^{*}\right) \mathrm{TB}-\mathrm{N}\left(^{*}\right)}$ was higher for the chiral dimers than their racemic counterparts, in accord with Meyer's model. ${ }^{18}$

The preparation of supramolecular mesogens via hydrogen bonding is a flexible and efficient alternative to covalent bonding, and a convenient way of adding functionality to a molecule in an effective and controllable manner. ${ }^{19}$ This is especially useful for introducing chirality to a system as it avoids the risk of partial racemisation during the synthetic process. An early example of supramolecular liquid crystal systems was reported in the 1950s by Jones and Gray, who described the thermal behaviour of the 4-alkoxybenzoic acids. The mesogenic behaviour was attributed to the formation of calamitic-like, symmetric homomeric complexes of two equivalent benzoic acid fragments with a supramolecular core formed via hydrogen bonding. ${ }^{20}$ This approach was extended in 2015 to the formation of the first supramolecular twist-bend nematogen CB6OBA (see Table 1), in which two acid dimers hydrogen-bond to form a bent supramolecular trimer. ${ }^{21}$

We have previously reported the thermal behaviour of equimolar mixtures containing the hydrogen bond acceptors: 10B6OS, CB6OS and nOS with different lengths of terminal alkoxy chain, $n$, and the hydrogen bond donors, the extensively studied 4-alkoxybenzoic acids, $n$ OBAs, and CB6OBA, the structures of which are shown in Table $1 .{ }^{22-24}$ These complexes were the first examples of how molecular recognition between unlike $\mathrm{H}$-bond donor and acceptor fragments can induce spontaneous chirality in a condensed phase, and formed the twist-bend nematic phase despite the components being either nonmesogenic (1OB6OS, CB6OS, nOS) or exhibiting only conventional nematic or smectic phases (nOBAs). The hydrogen bond between pyridyl-based fragments (S) and benzoic acids (BA) is strong enough to promote liquid crystalline complexes ${ }^{25}$ and the molecular curvature required for $\mathrm{N}_{\mathrm{TB}}$ phase behaviour is imparted by the flexible hexyloxy (6O) spacer of the longer fragment. ${ }^{26}$

Here, we combine our investigations on chiral and supramolecular twist-bend nematogens, and report the effect of molecular chirality in hydrogen-bonded mixtures containing a chiral branched terminal chain in place of the alkyl chains $(n)$ in the nOBA and nOS series, specifically $(S)$-2-methylbutyl, which is denoted herein as $(S)-(2-\mathrm{Me}) 4$. The structures of the $\mathrm{H}$-bonded heterocomplexes formed in these mixtures are also illustrated in Table 1.

\section{Experimental}

\section{Synthesis and analytical data}

1OB6OS, ${ }^{24} \mathrm{CB} \mathrm{OS}^{22}$ and CB6OBA ${ }^{21,23}$ were synthesised by methods reported elsewhere. The alcoholic stilbazole 4-[(E)-2-(pyridin-4yl)ethenyl]phenol (HOS) was prepared through the base-catalysed condensation of 4-hydroxybenzaldehyde and 4-methylpyridine. ${ }^{27}$ This was combined with the relevant bromoalkane in a Williamson ether synthesis to give the alkoxy-terminated stilbazole compounds 4OS and (S)-(2-Me)4OS. ${ }^{23}$ Butoxybenzoic acid was purchased from Sigma Aldrich and purified for use in mixtures by recrystallisation from ethanol $\left(10 \mathrm{~mL} \mathrm{~g}^{-1}\right)$. $(S)$-2-methylbutoxybenzoic acid

Table 1 Hydrogen bond acceptors and hydrogen bond donors used in this work, and the heterocomplexes formed. '1OB' denotes a biphenyl mesogenic unit terminated with a methoxy group; 'CB' a cyanobiphenyl moiety; '6O' a hexyloxy flexible spacer; ' $\mathrm{S}$ ' a 4-stilbazole unit; 'BA' a benzoic acid moiety; '(S)-(2-Me)4O' a 2 -methylbutoxy chain and '4O' a butoxy terminal chain
H-bond acceptors
H-bond donors

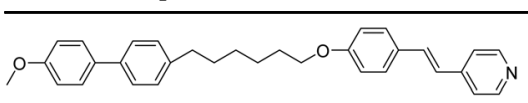

$1 \mathrm{OB} 6 \mathrm{OS}$

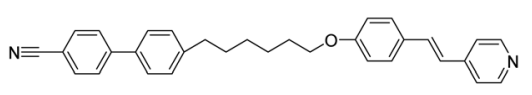

CB6OS

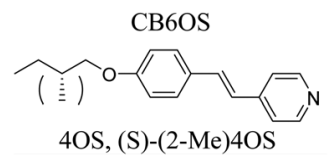

Heterocomplexes formed by H-bonding
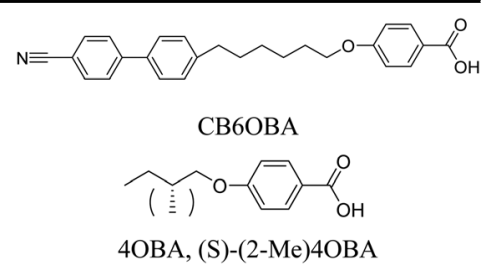
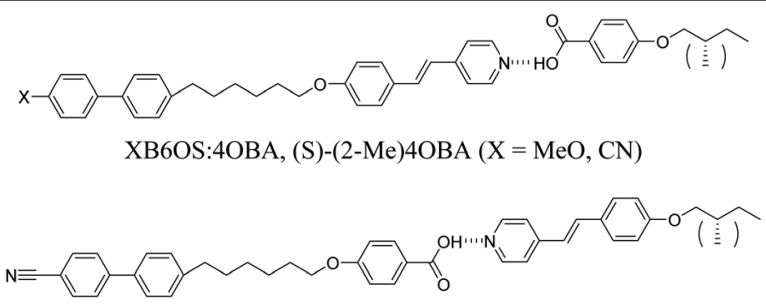

CB6OBA:4OS, (S)-(2-Me)4OS 
$((S)-(2-\mathrm{Me}) 4 \mathrm{OBA})$ was synthesised by a standard method reported elsewhere. ${ }^{22,28}$ Analytical data are given in the ESI. $\dagger$

\section{Polarised optical microscopy (POM)}

POM measurements were performed using an Olympus BH2 polarising light microscope equipped with a Linkam TMS 92 hot stage (University of Aberdeen) or a Zeiss Axio Imager A2m polarizing microscope equipped with a Linkam heating stage (University of Warsaw). Glass cells obtained from Warsaw Military University of Technology (WAT) with thicknesses of 1.6 or 3 microns, with ITO transparent electrodes and polymer aligning layers were used.

\section{Differential scanning calorimetry (DSC)}

The thermal behaviour of the final products and the binary mixtures was investigated using a Mettler Toledo DSC1 differential scanning calorimeter (University of Aberdeen) equipped with a TSO 801RO sample robot and calibrated using indium and zinc standards. The temperature scan profile in all cases, unless otherwise stated, was heat, cool and reheat at $10 \mathrm{~K} \mathrm{~min}^{-1}$ under nitrogen, with a 3-minute isotherm between heating and cooling segments. Thermal data were extracted from the second heating trace unless otherwise stated.

\section{X-Ray diffraction (XRD)}

Small-angle X-ray diffraction (SAXS) patterns for powder samples were obtained with a Bruker Nanostar system using $\mathrm{CuK} \alpha$ radiation and patterns were collected with an area detector VANTEC2000 (University of Warsaw). The temperature of the sample was controlled with a precision of $\pm 0.1 \mathrm{~K}$. Samples were prepared either in thin-walled glass capillaries or as droplets on a heated surface. Wide-angle diffractograms (WAXS) were obtained with a Bruker D8 GADDS system ( $\mathrm{CuK} \alpha$ line, Goebel mirror, point beam collimator, VANTEC2000 area detector) (University of Warsaw).

\section{Binary/ternary mixtures}

Binary and ternary mixtures were prepared by co-dissolving preweighed amounts of each compound in dichloromethane or chloroform and allowing the solvent to evaporate slowly at room temperature. The mixtures were further dried in a vacuum oven at $50{ }^{\circ} \mathrm{C}$ for $\sim 16-20 \mathrm{~h}$. Binary phase diagrams were constructed and, where necessary, used to estimate a virtual temperature for transitions precluded from direct observation by crystallisation on cooling, by extrapolation of the relevant trendline.

\section{Results and discussion}

\section{OBA and $(S)-(2-\mathrm{Me}) 40 B A$}

The chiral alkoxybenzoic acid, $(S)-(2-\mathrm{Me}) 4 \mathrm{OBA}$ did not show liquid crystalline behaviour. However, on heating from the crystalline solid a reversible transition to a disordered, or "soft" crystal phase (SCr) was seen prior to clearing. In order to first predict virtual mesogenic behaviour for the chiral acid, and also assess the effects of a chiral branch on the liquid crystallinity of supramolecular systems, binary mixtures of the nematogenic
Table 2 Transition temperatures $\left(T,{ }^{\circ} \mathrm{C}\right)$ and associated enthalpy changes $\left(\Delta H, \mathrm{~kJ} \mathrm{~mol}{ }^{-1}\right)$ for the 4OBA:(S)-(2-Me)4OBA binary mixtures

\begin{tabular}{|c|c|c|c|}
\hline mol\% $(S)-(2-\mathrm{Me}) 4 \mathrm{OBA}$ & MP & SCr & $\mathrm{N}^{*}$ \\
\hline $100(S)-(2-\mathrm{Me}) 4 \mathrm{OBA}$ & $105(3.55)$ & $108(12.44)$ & - [106] \\
\hline 90 & 96 (18.31) & $110(12.81)$ & - $110(3.10)$ \\
\hline 80 & $86(18.18)$ & $110(10.51)$ & $117(3.07)$ \\
\hline 70 & $81(18.06)$ & 109 (10.56) & $122(3.05)$ \\
\hline 60 & $114(12.77)$ & $111^{a}(10.92)$ & $130(3.03)$ \\
\hline 50 & $114(14.05)$ & $113^{a}(10.85)$ & $134(3.01)$ \\
\hline 40 & $124(14.92)$ & $117^{a}(11.00)$ & $141(2.33)$ \\
\hline 30 & 133 (11.98) & & $146(2.43)$ \\
\hline 20 & $138(15.01)$ & • & $151(2.58)$ \\
\hline 10 & $144(17.31)$ & & $156(2.93)$ \\
\hline 0 (4OBA) & $147(17.87)$ & & - $160(2.64)$ \\
\hline
\end{tabular}

$\square$ denotes a virtual temperature. ${ }^{a}$ Temperature obtained from the DSC cooling trace.

non-chiral $4 \mathrm{OBA}^{29,30}$ and chiral $(S)-(2-\mathrm{Me}) 4 \mathrm{OBA}$ were prepared and their thermal behaviour is summarised in Table 2 along with those of the pure acids. For all mixtures containing chiral acid, the chiral nematic phase was seen in place of the conventional nematic, identifiable by its characteristic oily streak texture, shown in Fig. 1. For mixtures containing over $40 \mathrm{~mol} \%$ of $(S)-(2-\mathrm{Me}) 4 \mathrm{OBA}$, the same soft crystal phase ( $\mathrm{SCr}$ ) is also present, initially visible only by supercooling the chiral nematic phase (40-60 mol\% $(S)-(2-\mathrm{Me}) 4 \mathrm{OBA})$ but enantiotropic for $>60 \mathrm{~mol} \%$. The almost linear dependence of the $T_{\mathrm{SCrN}^{*}}$ line on the binary phase diagram, see Fig. 2, indicates that there is good miscibility of the mixture components in the SCr phase, contrary to the behaviour normally observed in conventional crystal phases.

These data are collected in a phase diagram in Fig. 2. $T_{\mathrm{N}\left({ }^{*}\right) \mathrm{I}}$ varies in essentially a linear manner on varying the composition of the mixture, and decreases rather steeply on increasing the concentration of $(S)$-(2-Me)4OBA - dropping by over $50 \mathrm{~K}$ between the two pure acids. The $T_{\mathrm{N}(*) \mathrm{I}}$ trendline was extrapolated to $100 \mathrm{~mol} \%(S)-(2-\mathrm{Me}) 4 \mathrm{OBA}$ to estimate a virtual $T_{\mathrm{N}^{*} \mathrm{I}}$ of $106{ }^{\circ} \mathrm{C}$ for the chiral acid. This behaviour is in accord with the change in $T_{\mathrm{N}(*) \mathrm{I}}$ seen on comparing other branched and unbranched molecular pairs and is attributed to the methyl branch disrupting the interactions between the mesogenic units.

\section{B6OS:4OBA/(S)-(2-Me)4OBA}

The bent-shaped, supramolecular complex formed by $10 \mathrm{OB} 6 \mathrm{OS}$ and 4OBA exhibits conventional nematic and twist-bend nematic phases. A third mesophase is observed below the $\mathrm{N}_{\mathrm{TB}}$ phase,
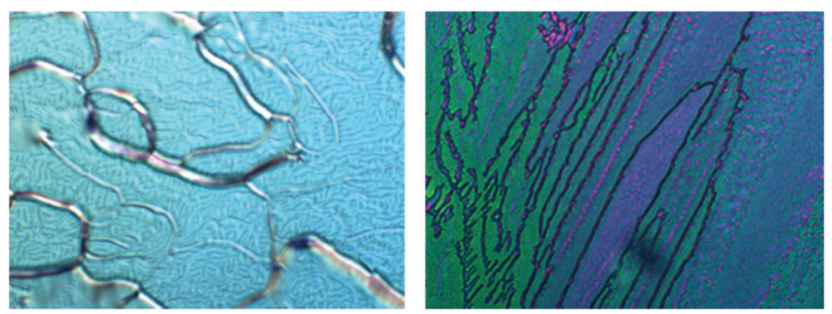

Fig. 1 POM textures obtained for a 40:60 4OBA: (S)-(2-Me)4OBA mixture in the chiral nematic phase (fingerprint and oily streak texture) (left) and soft crystal phase (right). 


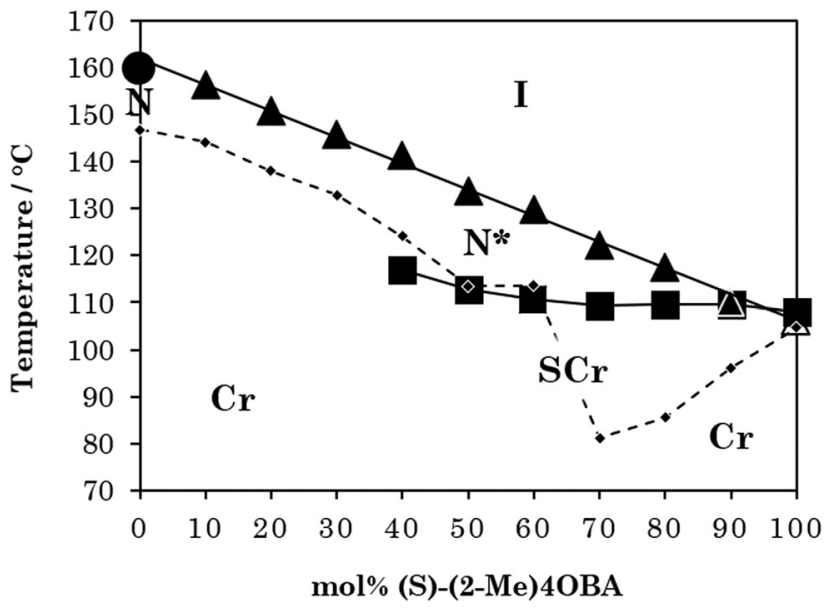

Fig. 2 Phase diagram of binary mixtures of 4OBA and (S)-(2-Me)4OBA,

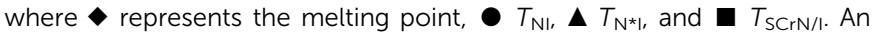
unfilled triangle represents a virtual transition temperature.

denoted the SmX phase; its monotropic nature precluded detailed analysis but it was suggested to be a highly ordered smectic phase. ${ }^{22,24}$ An equimolar mixture of the chiral benzoic acid, (S)-(2-Me)4OBA - here considered to be the chiral "analogue" of 4OBA - with 1 OB6OS does not form the $\mathrm{N}_{\mathrm{TB}}$ phase, instead a $\mathrm{SmA}^{*}$ phase is observed below the $\mathrm{N}^{*}$ phase. These assignments are made based on textural observations, an oily streak texture and focal conic fans in coexistence with homeotropic regions, characteristic of the chiral nematic and smectic $A^{*}$ phases, respectively. In order to assess the effects of the branch on the phases present in the 1OB6OS:4OBA mixture, a series of ternary mixtures were prepared containing the H-bond acceptor 1OB6OS and both $\mathrm{H}$-bond donor acids. The concentration of $1 \mathrm{OB} 6 \mathrm{OS}$ was kept constant at $50 \mathrm{~mol} \%$ and the relative amounts of $4 \mathrm{OBA}$ and $(S)-(2-\mathrm{Me}) 4 \mathrm{OBA}$ were varied to maintain the remaining $50 \mathrm{~mol} \%$, keeping the total numbers of pyridyl and acid groups equal. The transitional properties of these mixtures are given in Table 3.
Textural features similar to those found in a covalentlybonded $\mathrm{N}^{*}$ тв system were also observed in mixtures with less than $50 \%$ of the chiral component. Specifically, in a $3 \mu \mathrm{m}$ planar aligned cell, the oily streak texture of the chiral nematic phase transforms to a stripe pattern some degrees prior to the transition to the $\mathrm{N}^{*}$ тв phase, see Fig. 3. This phenomenon was observed in the $\mathrm{N}^{*}$ phase of both chirally-doped bent core materials and chiral twist-bend nematogens, and suggested to be a consequence of different modulated equilibrium director structures. ${ }^{18,31}$ In planar aligned cells, the helical axis of the $\mathrm{N}^{*}$ phase is oriented perpendicular to the substrate, and the free energy of this system is minimised when the cell thickness, $d$, is a multiple of the half pitch, $p / 2$. Since the pitch is temperature dependent, at most temperatures this condition is not fulfilled, and thus to minimise the free energy the helix becomes distorted. In a normal $\mathrm{N}^{*}$ system, these deformations typically involve the twist elastic constant $K_{22}$. However, in the system studied here we presume the bend elastic constant $K_{33}$ is smaller than $K_{22}$ and instead we see bend deformations, resulting in this stripe texture. The reason for this is not clear but may reflect the instantaneous formation of short pitch helices which influence the macroscopic bend elastic constant. ${ }^{32}$ At the $\mathrm{N}^{*}-\mathrm{N}^{*}$ тв transition, the oily streak pattern undergoes dynamic textural changes before settling into a generally uniform texture of birefringence and pattern akin to that seen in covalently-bonded chiral twist-bend nematogens. ${ }^{18}$ However, the narrow temperature range of the $\mathrm{N}^{*}$ тв phase presumably prevented further development of the more detailed stripe and chain-like textures seen previously in the $\mathrm{N}^{*}$ тв phase. ${ }^{18}$ This is the first observation of the chiral twist-bend nematic phase in a hydrogen-bonded system. In the smectic $A^{*}$ phase, characteristic focal conic fans were clearly observed when the phase was viewed between untreated glass slides (Fig. 3).

The phase diagram constructed from these data is shown in Fig. 4. It is clear that, as with the rod-like acid complexes (Fig. 2), $T_{\mathrm{N}(*) \mathrm{I}}$ is significantly reduced on switching from a straight to a

Table 3 Transition temperatures $\left(T,{ }^{\circ} \mathrm{C}\right)$ and associated enthalpy changes $\left(\Delta H, \mathrm{~kJ} \mathrm{~mol}^{-1}\right)$ for the $1 \mathrm{OB} 6 \mathrm{OS}: 4 \mathrm{OBA} /(\mathrm{S})-(2-\mathrm{Me}) 4 \mathrm{OBA}$ ternary mixtures

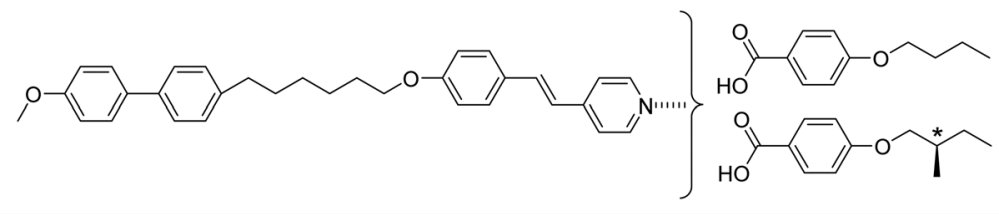

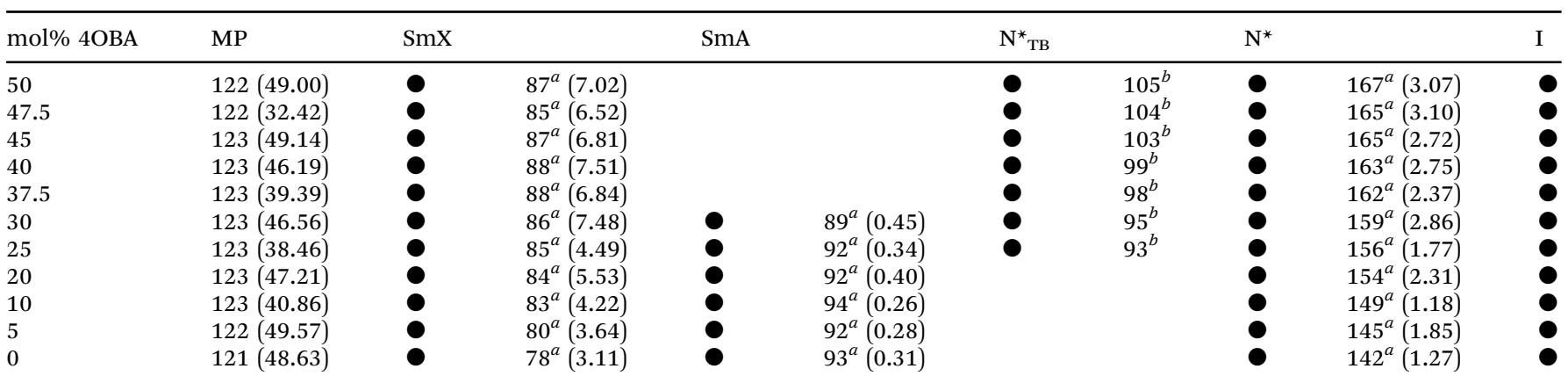

${ }^{a}$ Temperature obtained from the DSC cooling trace. ${ }^{b}$ Temperature obtained using POM. 

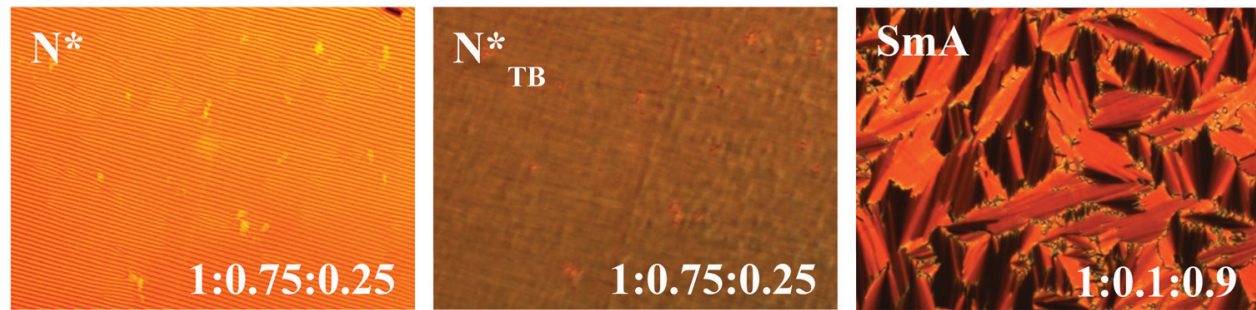

Fig. 3 POM images for 1OB6OS: 4OBA: (S)-(2-Me)4OBA with composition 1: $0.75: 0.25$ in the $\mathrm{N}^{*}$ and $\mathrm{N}^{*}$ TB phases (left and middle, respectively) in a 3 micron planar aligned cell, and with composition 1: 0.1:0.9 in the SmA phase (right) in untreated glass slides.

branched chain acid. The magnitude of this reduction is approximately half that seen for the acid dimers across Table 2, consistent with the presence of one methyl branch in 1OB6OS: $(S)-(2-\mathrm{Me}) 4 \mathrm{OBA}$ as opposed to two in the pure acid complex $(S)-(2-\mathrm{Me}) 4 \mathrm{OBA} . \quad T_{\mathrm{N}(*) \operatorname{TBN}(*)}$ also decreases with increasing amount of $(S)-(2-\mathrm{Me}) 4 \mathrm{OBA}$, and at $30 \mathrm{~mol} \%$ chiral acid in the mixture, the $\mathrm{N}^{*}$ тв phase is precluded by the emergence of a $\mathrm{SmA}^{*}$ phase. $T_{\mathrm{SmA}^{*} \mathrm{~N}^{*}}$ is reduced upon addition of the unbranched acid, but this is a weaker dependence. The transition temperatures of the SmX phase remain essentially constant across all mixture compositions.

\section{CB6OS:(S)-(2-Me)4OBA}

The supramolecular complex formed by CB6OS and 4OBA also exhibits conventional nematic and twist-bend nematic phases. ${ }^{22}$ The CB6OS:(S)-(2-Me)4OBA binary mixture exhibits the chiral nematic phase and, unlike its methoxy analogue 1OB6OS:(S)(2-Me)4OBA, the chiral twist-bend nematic phase. The ordered smectic phase (SmX) seen for 1OB6OS:(S)-(2-Me)4OBA is absent for CB6OS:(S)-(2-Me)4OBA. In a 3 micron planar aligned cell, the oily streak texture of the $\mathrm{N}^{*}$ phase again undergoes a dynamic change at the $\mathrm{N}^{*}-\mathrm{N}^{*}$ тв phase transition, to a blocky, somewhat striped texture with areas of chain-like defects, see Fig. 5, also similar to textures reported for covalently bonded

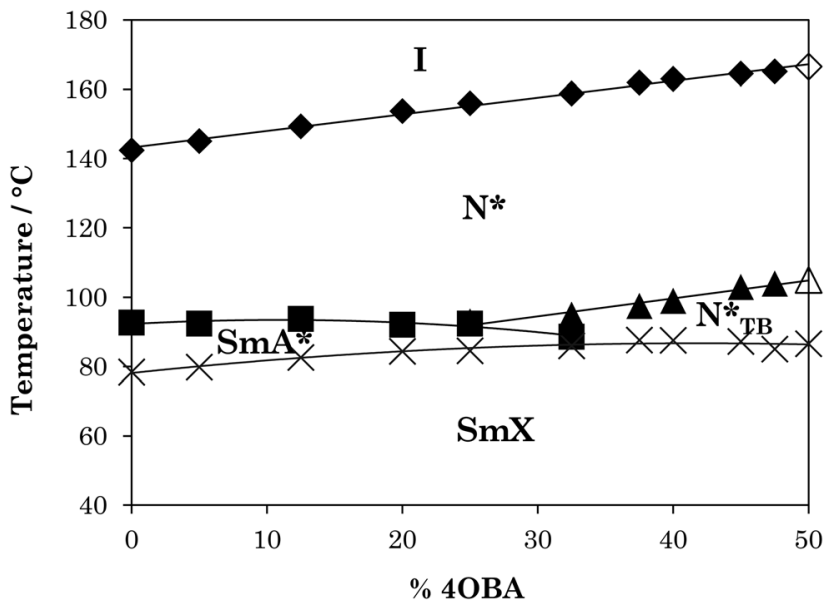

Fig. 4 Dependence of phase behaviour of the 1OB6OS:4OBA:(S)(2-Me)4OBA series of ternary mixtures on the molar percentage of achiral $\mathrm{H}$-bond donor 4OBA, where represents $T_{\mathrm{N}^{*},}, T_{\mathrm{SmAN}^{*},} \boldsymbol{\Delta} T_{\mathrm{N}^{*} \mathrm{TBN}}$ and $\times$ $T_{\text {NTB/SmA-Smx }}$. Unfilled diamond and triangle represent conventional $\mathrm{N}-1$ and $\mathrm{N}_{\mathrm{TB}}-\mathrm{N}$, respectively. Melting points omitted for clarity. chiral twist-bend nematogens. ${ }^{18}$ The X-ray patterns for both the $\mathrm{N}^{*}$ and $\mathrm{N}^{*}$ тв phases are also shown in Fig. 5 and are very similar: specifically, these contain diffuse wide-angle scattering indicative of the liquid-like ordering in nematic phases. There is no visible small-angle reflection at the short scan times used ( $\sim 10$ minutes exposure time, as the sample was prone to crystallisation), and hence the local arrangement of the molecules unfortunately could not be determined.

The transition temperatures and associated enthalpy changes for the mixtures are given in Table 4 . The methyl branch lowers the $T_{\mathrm{N}(*) \mathrm{I}}$ by $22 \mathrm{~K}$, approximately the same as in the $1 \mathrm{OB} 6 \mathrm{OS}$ mixtures, and this is accompanied by a reduction in $\Delta S_{\mathrm{N}(*) \mathrm{r}}$. These observations are consistent with the behaviour of unbranched and branched pairs of covalently bonded dimers, and suggests an increase in biaxiality in the $\mathrm{N}^{*}$ phase on branching the complex. ${ }^{33,34} T_{\mathrm{N}\left(^{*}\right) \operatorname{TBN}(*)}$ is less affected, lowered by only $7 \mathrm{~K}$ on chain branching, suggesting that a reduction in interaction strength parameter has a lesser effect on the stability of the $\mathrm{N}^{*}$ тв phase than on that of the $\mathrm{N}^{*}$ phase, and highlighting the importance of molecular curvature for the predominantly shapedriven $\mathrm{N}_{\mathrm{TB}}-\mathrm{N}$ transition. ${ }^{35}$ In previous studies we have also noted that $\mathrm{N}^{*} \mathrm{~TB}^{-} \mathrm{N}^{*}$ transition temperatures for optically pure chiral dimers have been found to be higher than their racemic counterparts ${ }^{18}$ in accord with the elastic instability model described by Meyer.$^{13}$ While we have no racemic counterpart for comparison here, it is possible that such an enhancement in $T_{\mathrm{N}^{*} \mathrm{TBN}^{*}}$ has somewhat offset the effects of the branch, resulting in a smaller reduction than expected.

(a)
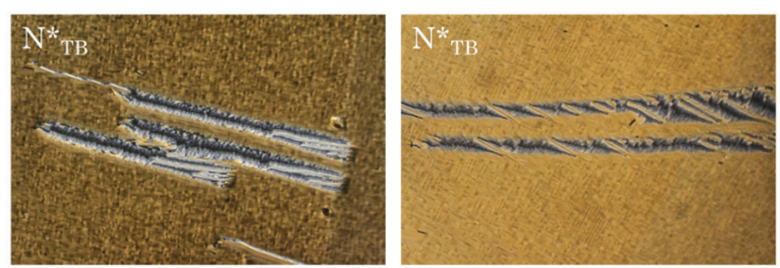

(b)
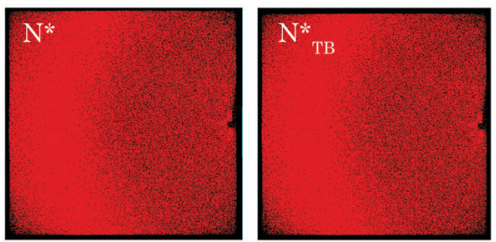

Fig. 5 (a) POM textures seen for the chiral twist-bend nematic phase and (b) X-ray diffraction patterns obtained in the $\mathrm{N}^{*}$ and $\mathrm{N}^{*}$ TB phases for CB6OS:(S)-(2-Me)4OBA. 
Table 4 Transition temperatures $\left(T,{ }^{\circ} \mathrm{C}\right)$ and associated enthalpy changes $\left(\Delta H, \mathrm{~kJ} \mathrm{~mol}^{-1}\right)$ for the CB6OS:4OBA and CB6OS:(S)-(2-Me)4OBA supramolecular dimers

\begin{tabular}{lllllll}
\hline & MP & $\mathrm{N}^{*}$ тв & & $\mathrm{N}^{*}$ & & $\mathrm{I}$ \\
\hline 4OBA & $107(29.67)$ & $\bullet$ & $95^{b}$ & $\bullet$ & $167(2.87)$ & $\bullet$ \\
$(2-\mathrm{Q})$ 4OBA & $103(44.26)$ & $\bullet$ & $88^{a}(0.14)$ & $\bullet$ & $145(1.27)$ & $\bullet$
\end{tabular}

${ }^{a}$ Temperature obtained from the DSC cooling trace. ${ }^{b}$ Temperature obtained using POM.

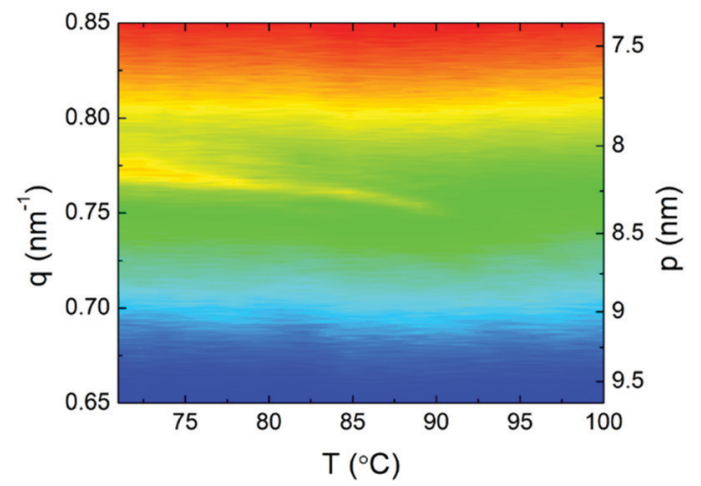

Fig. 6 RSoXS pattern obtained for CB6OS:(S)-(2-Me)4OBA.

Finally, resonant soft X-ray scattering (RSoXS), with incident beam energy tuned to the absorption K-edge of carbon was used to confirm the helical structure of the chiral twist-bend nematic phase. A weak, single resonant signal was found in the $\mathrm{N}^{*}$ тв phase (Fig. 6), corresponding to periodicity 8.1-8.4 nm approximately 2 complex lengths. Interestingly, the observed temperature dependence of the helical pitch near the $\mathrm{N}^{*} \mathrm{~TB}-\mathrm{N}^{*}$ transition is much weaker than typically seen for twist-bend nematic phases in covalently-bonded dimers, suggesting a strongly first-order transition, although the enthalpy change associated with the $\mathrm{N}^{*} \mathrm{~TB}-\mathrm{N}^{*}$ transition remains characteristically small.

\section{CB6OBA:(S)-(2-Me)4OS}

The supramolecular complex CB6OBA:4OS is an enantiotropic nematogen, and on cooling also exhibits the $\mathrm{N}_{\mathrm{TB}}$ phase. ${ }^{23}$ Unlike in the 1OB6OS:4OBA and CB6OS:4OBA systems, this is followed on further cooling by a SmC phase and another more ordered smectic phase, which here we denote $\mathrm{SmX}_{2}$. The use of a chiral molecule as the hydrogen bond acceptor - $(S)-(2-\mathrm{Me}) 4 \mathrm{OS}$, here considered as the chiral "equivalent" of $4 \mathrm{OS}$ - to form the chiral complex CB6OBA:(S)-(2-Me)4OS does not alter the phase behaviour; on cooling from the isotropic phase we observe the sequence $\mathrm{N}^{*}-\mathrm{N}^{*}{ }_{\mathrm{TB}}-\mathrm{SmC}^{*}-\mathrm{SmX}_{2}{ }^{*}$. The transition temperatures and associated enthalpy changes for both complexes are given in Table 5.

The highest temperature phase is a conventional chiral nematic phase $\mathrm{N}^{*}$, assigned by observation of a characteristic oily streak texture, see Fig. 7. Cooling from the $\mathrm{N}^{*}$ phase led to the dynamic $\mathrm{N}^{*}-\mathrm{N}^{*}$ тв textural change as seen in previous systems showing the $\mathrm{N}^{*}$ тв phase, and a resulting texture containing rope- and chain-like defects (Fig. 7). X-ray diffraction patterns of these higher temperature phases, also shown in Fig. 7, exhibited only diffuse wide- and small-angle signals, consistent with the absence of a lamellar structure and confirm the liquid-like ordering of molecules in the nematic phases. A transition from the $\mathrm{N}^{*}$ тв phase to a smectic phase with liquidlike order within the layers is clear from the narrowing of the small-angle X-ray diffraction signal while the high-angle signal remains diffuse. Optically, this phase retains many of the features of the $\mathrm{N}^{*}$ тв phase, most significantly the stripe and

Table 5 Transition temperatures $\left(T,{ }^{\circ} \mathrm{C}\right)$ and associated enthalpy changes $\left(\Delta H, \mathrm{~kJ} \mathrm{~mol}^{-1}\right)$ for the CB6OBA:4OS and CB6OBA:(S)-(2-Me)4OS supramolecular dimers

\begin{tabular}{|c|c|c|c|c|c|c|c|c|}
\hline & MP & $\mathrm{SmX}^{*}$ & & $\mathrm{SmC}^{*}$ & $\mathrm{~N}^{*}{ }_{\mathrm{TB}}$ & & $\mathrm{N}^{*}$ & \\
\hline
\end{tabular}

${ }^{a}$ Temperature obtained from the DSC cooling trace. ${ }^{b}$ Temperature obtained using POM.
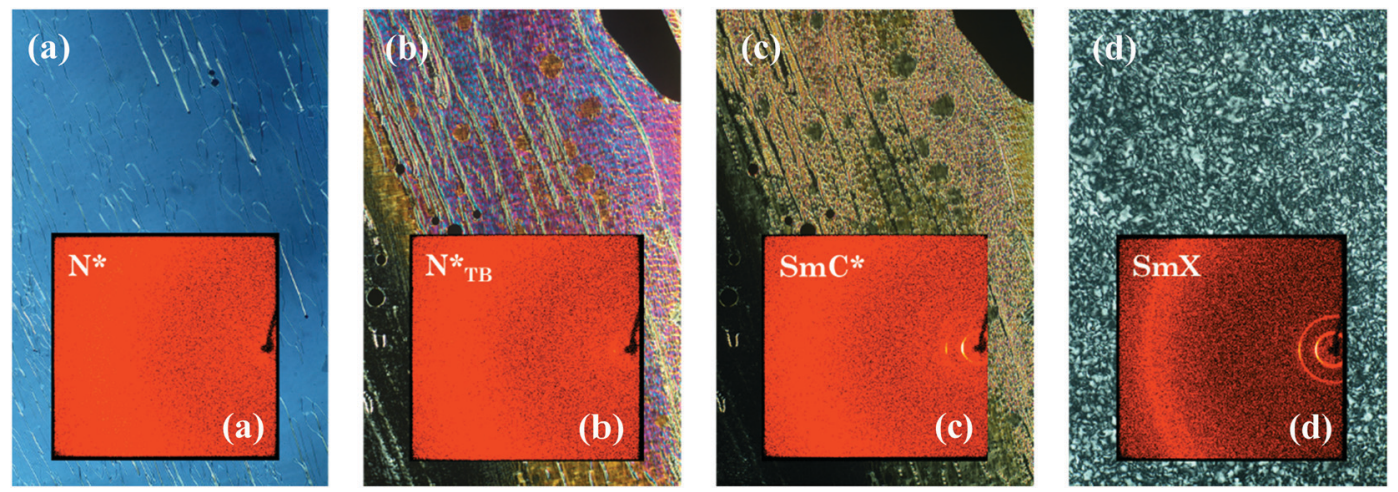

Fig. 7 POM images and associated X-ray diffraction patterns (insets) for CB6OBA:(S)-(2-Me)4OS in the (a) $\mathrm{N}^{*}$, (b) $\mathrm{N}^{*} \mathrm{~TB}$, (c) SmC* and (d) SmX phases. 


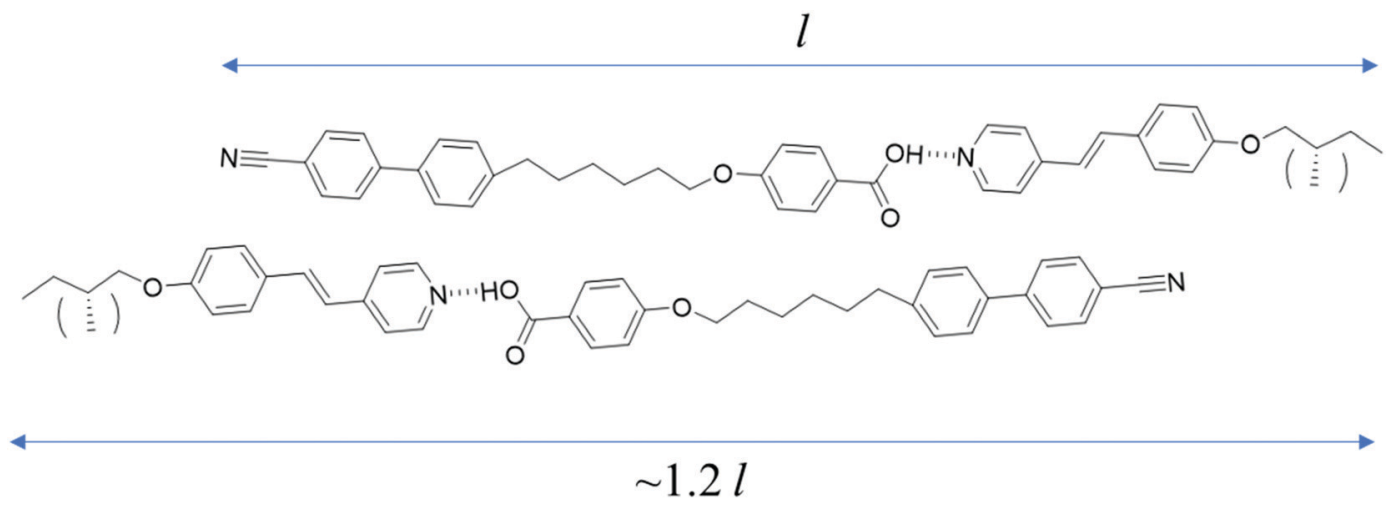

Fig. 8 Proposed molecular arrangement in the lamellar phases of CB6OBA:(S)-(2-Me)4OS.

Table 6 Layer thickness in the smectic phases obtained from integrated diffraction signals for the CB6OBA:(S)-(2-Me)4OS complex

\begin{tabular}{llllll}
\hline & & $\begin{array}{l}\text { Complex } \\
\text { length, } l / \AA\end{array}$ & $\begin{array}{l}\text { Low angle } \\
\text { signals/A }\end{array}$ & $\begin{array}{l}\text { High angle } \\
\text { signals/A }\end{array}$ & $d / l$ \\
\hline PB6OBA:(S)-(2- & SmC $^{*}$ & 40.1 & $47.8,24.2,13.0$ & 4.3 & 1.2 \\
Me)4OS & SmX & & $46.5,23.5$ & $4.6,4.2$ & 1.2
\end{tabular}

rope-like pattern, although these "ropes" now appear to have a more braided texture. Homeotropically aligned areas which appear black in the preceding $\mathrm{N}^{*}$ тв phase also remain as such in the smectic phase. Given the textural similarities of this phase to the higher temperature smectic phase seen in analogous achiral complexes, it has been tentatively assigned as a $\mathrm{SmC}^{*}$ phase. We note the possibility that the SmC phase reported for the achiral complexes may belong to the new class of heliconical smectic phases formed by achiral materials, ${ }^{36,37}$ but its monotropic nature precluded further investigation. ${ }^{23}$ It is therefore possible that the helical structure of the $\mathrm{SmC}^{*}$ phase seen here is not conventional, and this may be a subject of future work. On further cooling a bright, schlieren-like mosaic texture emerges from the dark regions of the $\mathrm{SmC}^{*}$ phase, and this strongly resembles the texture of the lowest temperature phase exhibited by the achiral CB6OBA:nOS complexes with $n=3-10$. The X-ray diffraction pattern retains sharp small-angle signals, and the high angle signal is split and narrowed, suggesting a tilted hexatic-type smectic phase. This shows that the SmX phases present in the CB6OBA: $n$ OS series $(n=3-10)$ and CB6OBA:( $S)-(2-\mathrm{Me}) 4 \mathrm{OS}$ are the same, and we have denoted the latter $\mathrm{SmX}^{*}$ as it is formed from chiral molecules. Across all these phases, the small angle signals appear at slightly more than the full complex length $(d / l \sim 1.2 ; d$ is the periodicity deduced from the position of the lowest angle diffraction signal, $l$ is the estimated length of the molecule in the most stretched conformation), hence the arrangement of the complexes may be partially bilayer with strong interdigitation, see structure in Fig. 8 and data in Table 6.

Branching the terminal chain has the expected effect on $T_{\mathrm{N}^{*} \mathrm{I}}$, specifically a reduction by $29 \mathrm{~K}$. The branch also decreases $T_{\mathrm{N}\left({ }^{*}\right) \mathrm{TBN}\left({ }^{*}\right)}$, but as in the previous systems, it is a smaller reduction of only $2 \mathrm{~K}$. The opposite effect is observed for the smectic phases, with both $\mathrm{SmC}^{*}$ and $\mathrm{SmX}^{*}$ increasing in stability with the branch. This enhancement of the smectic phases may be attributed to the branched group effectively filling void space in the tilted lamellar structure to such an extent that this stabilisation more than offsets the dilution in lateral interactions of the mesogenic cores brought about by the branch. This is also evident in the increased melting point of the branched complex, suggesting a more efficient packing in the crystal phase compared with that for the unbranched one.

\section{Conclusion}

We have described the first examples of the chiral twist-bend nematic phase, $\mathrm{N}^{*} \mathrm{~TB}$, exhibited by supramolecular liquid crystals formed by hydrogen-bonding between unlike $\mathrm{H}$-bonded donors and acceptors. In general, the properties of the $\mathrm{N}^{*}$ тв phase strongly resemble those previously observed in covalently bonded materials. In particular, the optical textures observed are distinct and contain unusual features, the physical significance of which remain unclear. In addition, the thermal stability of the $\mathrm{N}^{*}$ тв phase is greater than that of the conventional $\mathrm{N}_{\mathrm{TB}}$ phase exhibited by achiral materials. We have observed a transition between the $\mathrm{N}^{*}$ тв phase and a $\mathrm{SmC}^{*}$ phase, and we have still to determine whether the structure of this $\mathrm{SmC}^{*}$ phase is conventional, or more akin to the heliconical smectic phases recently reported for achiral molecules with a strikingly short helical pitch. ${ }^{36,37}$

\section{Funding}

The work was supported by the National Science Centre (Poland) under the grant no. 2016/22/A/ST5/00319.

\section{Conflicts of interest}

The authors declare no conflict of interest.

\section{Acknowledgements}

RW gratefully thanks the Carnegie Trust for the Universities of Scotland for the award of a PhD Scholarship (2015-2018). 


\section{References}

1 M. Cestari, et al., Phase behavior and properties of the liquid-crystal dimer $1^{\prime \prime}, 7^{\prime \prime}$-bis(4-cyanobiphenyl-4'-yl) heptane: A twist-bend nematic liquid crystal, Phys. Rev. E: Stat., Nonlinear, Soft Matter Phys., 2011, 84, 031704.

2 R. B. Meyer, Structural problems in liquid crystal physics, Les Houches Summer School in Theoretical Physics, Molecular Fluids, Gordon and Breach, 1976, pp. 316-320.

3 I. Dozov, On the spontaneous symmetry breaking in the mesophases of achiral banana-shaped molecules, Europhys. Lett., 2001, 56, 247-253.

$4 \mathrm{~V}$. Borshch, et al., Nematic twist-bend phase with nanoscale modulation of molecular orientation, Nat. Commun., 2013, 4, 2635.

5 P. A. Henderson and C. T. Imrie, Methylene-linked liquid crystal dimers and the twist-bend nematic phase, Liq. Cryst., 2011, 38, 1407-1414.

6 E. Cruickshank, et al., Sulfur-linked cyanobiphenyl-based liquid crystal dimers and the twist-bend nematic phase, Liq. Cryst., 2019, 46, 1595-1609.

7 D. A. Paterson, J. P. Abberley, W. T. A. Harrison, J. Storey and C. T. Imrie, Cyanobiphenyl-based liquid crystal dimers and the twist-bend nematic phase, Liq. Cryst., 2017, 44, 127-146.

8 D. A. Paterson, et al., The role of a terminal chain in promoting the twist-bend nematic phase: the synthesis and characterisation of the 1-(4-cyanobiphenyl-4'-yl)-6(4-alkyloxyanilinebenzylidene-4'-oxy)hexanes, Liq. Cryst., 2018, 45, 2341-2351.

9 R. J. Mandle, C. T. Archbold, J. P. Sarju, J. L. Andrews and J. W. Goodby, The dependency of nematic and twist-bend mesophase formation on bend angle, Sci. Rep., 2016, 6, 1-12.

10 M. Šepelj, et al., Intercalated liquid-crystalline phases formed by symmetric dimers with an $\alpha, \omega$-diiminoalkylene spacer, J. Mater. Chem., 2007, 17, 1154-1165.

11 R. J. Mandle, The dependency of twist-bend nematic liquid crystals on molecular structure: A progression from dimers to trimers, oligomers and polymers, Soft Matter, 2016, 12, 7883-7901.

12 W. D. Stevenson, et al., Dynamic calorimetry and XRD studies of the nematic and twist-bend nematic phase transitions in a series of dimers with increasing spacer length, Phys. Chem. Chem. Phys., 2018, 20, 25268-25274.

13 C. Meyer, Nematic twist-bend phase under external constraints, Liq. Cryst., 2016, 43, 2144-2162.

14 L. Longa and W. Tomczyk, Twist-bend nematic phase in the presence of molecular chirality, Liq. Cryst., 2018, 45, 2074-2085.

15 R. J. Mandle and J. W. Goodby, A novel nematic-like mesophase induced in dimers, trimers and tetramers doped with a high helical twisting power additive, Soft Matter, 2018, 14, 8846-8852.

16 C. T. Archbold, E. J. Davis, R. J. Mandle, S. J. Cowling and J. W. Goodby, Chiral dopants and the twist-bend nematic phase-induction of novel mesomorphic behaviour in an apolar bimesogen, Soft Matter, 2015, 11, 7547-7557.

17 E. Gorecka, et al., A Twist-Bend Nematic $\left(\mathrm{N}_{\mathrm{TB}}\right)$ Phase of Chiral Materials, Angew. Chem., Int. Ed., 2015, 54, 10155-10159.
18 R. Walker, J. M. D. Storey, C. T. Imrie, D. Pociecha and E. Gorecka, The Chiral Twist-Bend Nematic Phase $\left(\mathrm{N}^{*}{ }_{\mathrm{TB}}\right)$, Chem. - Eur. J., 2019, 25, 13329-13335.

19 C. M. Paleos and D. Tsiourvas, Supramolecular hydrogenbonded liquid crystals, Liq. Cryst., 2001, 28, 1127-1161.

20 G. W. Gray and B. Jones, Mesomorphism of some Alkoxynaphthoic Acids, Nature, 1951, 167, 83-84.

21 S. M. Jansze, A. Martínez-Felipe, J. M. D. Storey, A. T. M. Marcelis and C. T. Imrie, A twist-bend nematic phase driven by hydrogen bonding, Angew. Chem., Int. Ed., 2015, 54, 643-646.

22 R. Walker, et al., Hydrogen bonding and the design of twistbend nematogens, J. Mol. Liq., 2020, 303, 112630.

23 R. Walker, et al., Twist-Bend Nematogenic Supramolecular Dimers and Trimers Formed by Hydrogen Bonding, Crystals, 2020, 10, 175.

24 R. Walker, et al., Spontaneous chirality through mixing achiral components: A twist-bend nematic phase driven by hydrogen-bonding between unlike components, Chem. Commun., 2018, 54, 3383-3386.

25 T. Kato and J. M. J. Fréchet, New Approach to Mesophase Stabilization through Hydrogen-Bonding Molecular Interactions in Binary Mixtures, J. Am. Chem. Soc., 1989, 111, 8533-8534.

26 D. A. Paterson, et al., Understanding the twist-bend nematic phase: The characterisation of 1-(4-cyanobiphenyl-4'-yloxy)6-(4-cyanobiphenyl-4'-yl)hexane (CB6OCB) and comparison with CB7CB, Soft Matter, 2016, 12, 6827-6840.

27 S. El Sayed, et al., 2,4-Dinitrophenyl Ether-Containing Chemodosimeters for the Selective and Sensitive in Vitro and in Vivo Detection of Hydrogen Sulfide, Supramol. Chem., 2015, 27, 244-254.

28 J. Jensen, S. C. Grundy, S. L. Bretz and C. S. Hartley, Synthesis and characterization of self-assembled liquid crystals: P-alkoxybenzoic acids, J. Chem. Educ., 2011, 88, 1133-1136.

29 P. Painter, C. Cleveland and M. Coleman, An Infrared Spectroscopic Study of p-n-Alkoxybenzoic Acids, Mol. Cryst. Liq. Cryst. Sci. Technol., Sect. A, 2000, 348, 269-293.

30 A. Martínez-Felipe, A. G. Cook, M. J. Wallage and C. T. Imrie, Hydrogen bonding and liquid crystallinity of low molar mass and polymeric mesogens containing benzoic acids: A variable temperature Fourier transform infrared spectroscopic study, Phase Transitions, 2014, 87, 1191-1210.

31 S. M. Salili, et al., Spontaneously modulated chiral nematic structures of flexible bent-core liquid crystal dimers, Liq. Cryst., 2017, 44, 160-167.

32 D. Pociecha, et al., Critical behavior of the optical birefringence at the nematic to twist-bend nematic phase transition, Phys. Rev. E, 2018, 98, 052706.

33 C. T. Imrie, Laterally substituted dimeric liquid crystals, Liq. Cryst., 1989, 6, 391-396.

34 J. P. Abberley, et al., Structure-property relationships in twist-bend nematogens: the influence of terminal groups, Liq. Cryst., 2017, 44, 68-83. 
35 R. Walker, et al., Molecular curvature, specific intermolecular interactions and the twist-bend nematic phase: the synthesis and characterisation of the 1-(4-cyanobiphenyl4'-yl)-6-(4-alkylanilinebenzylidene-4'-oxy)hexanes (CB6O.m), Soft Matter, 2019, 15, 3188-3197.
36 M. Salamończyk, et al., Multi-level chirality in liquid crystals formed by achiral molecules, Nat. Commun., 2019, 10, 1922.

37 J. P. Abberley, et al., Heliconical smectic phases formed by achiral molecules, Nat. Commun., 2018, 9, 228. 